\title{
The measurement of $\alpha$ from the B-factories
}

\author{
A. J. BEVAN \\ Department of Physics, Queen Mary University of London, Mile End Road, London E1 4NS, \\ England.
}

\begin{abstract}
Significant progress toward measuring the CKM angle $\alpha$ has been made by the Bfactories over the past decade. This work has culminated in a constraint on $\alpha$ with a precision of less than $4^{\circ}$.
\end{abstract}

Keywords: CP Violation, CKM, $\alpha$

PACS: $13.25 \mathrm{Hw}, 12.39 . \mathrm{St}, 14.40 . \mathrm{Nd}$

\section{INTRODUCTION}

The Standard Model of Particle Physics (SM) accounts for $\mathrm{CP}$ violation via a single complex parameter in the Cabibbo-Kobayashi-Maskawa $3 \times 3$ quark-mixing matrix [1]. It is possible to write down six triangle relations using the unitarity of the CKM matrix. One of these, the so-called Unitarity Triangle, has internal angles $\alpha, \beta$, and $\gamma^{1}$, and these can be studied using decays of $B$ mesons. In these proceedings I summarize the measurement of $\alpha$, where $\alpha \equiv \arg \left[-V_{\mathrm{td}} V_{\mathrm{tb}}^{*} / V_{\mathrm{ud}} V_{\mathrm{ub}}^{*}\right]$, and the $V_{\mathrm{ij}}$ are CKM matrix elements for $i$ to $j$ quark mixing processes.

There are two B-Factories in existence, one at the SLAC National Laboratory, USA, and the other at KEK, Japan. Both are described elsewhere [2], and they collide beams of $e^{-}$and $e^{+}$with a center of mass $(\mathrm{CM})$ energy predominantly corresponding to the $\Upsilon(4 \mathrm{~S})$ resonance. BABAR and Belle have accumulated 425 and $771 \mathrm{fb}^{-1}$ of data at the $\Upsilon(4 \mathrm{~S})$, respectively. The beam energies are asymmetric, so that the $\mathrm{CM}$ is boosted with respect to the laboratory frame of reference. Most of the decay products of the $\Upsilon(4 \mathrm{~S})$ are $B-\bar{B}$ pairs. By studying combinations of the decays of neutral and charged $B$ mesons to $h h$ final states, where $h$ is a $\pi$ or $\rho$ meson, we can measure $\alpha$.

The first step in this measurement is to isolate signal candidates $\left(B_{\mathrm{REC}}\right)$. This is done by using two kinematic variables that are computed using the known beam energy in order to reduce resolution effects from experimental reconstruction, and the correlation between these variables. These are: $\Delta E$ the difference in reconstructed energy of the $B$ candidate and half of the total beam energy in the CM frame of reference; and $m_{\mathrm{ES}}$ which is an effective invariant mass of the $B$ candidate computed using the beam energy instead of the reconstructed $B$ candidate energy. Signal events peak at zero in the $\Delta E$ distribution, and at the $B$ mass in $m_{\mathrm{ES}}=5.28 \mathrm{GeV} / \mathrm{c}^{2}$.

Having identified the $B_{\text {REC }}$ the next step in the measurement involves computing the proper-time difference $\Delta t$ between the decay of the $B_{\text {REC }}$ and the other $B$ in the event

1 The notation $\phi_{1}, \phi_{2}$, and $\phi_{3}$ is used by the Belle Collaboration. 
( $\left.B_{\mathrm{TAG}}\right)$. In order to compute $\Delta t$ we use the Lorentz boost of the CM in the lab frame and the spatial separation between the reconstructed $B_{\text {REC }}$ and $B_{\mathrm{TAG}}$ vertices. It is important to account for the effect of detector resolution on $\Delta t$. This has several sources including the ability to reconstruct the $B_{\mathrm{REC}}$ and $B_{\mathrm{TAG}}$ vertices, and the decay in flight of fully or partially reconstructed intermediate particles in the $B_{\mathrm{REC}}$ or $B_{\mathrm{TAG}}$. The final ingredient required for a time-dependent $\mathrm{CP}$ measurement is to identify the flavor of the $B_{\mathrm{TAG}}$. This is done using a flavor tagging algorithm that is able to assign a $B^{0}$ or $\bar{B}^{0}$ flavor tag to $B_{\mathrm{TAG}}$ at the time it decayed with a probability that depends on the $B_{\mathrm{TAG}}$ final state. The complement of this is called the mistag probability of the event. As the $B^{0}-\bar{B}^{0}$ mixing frequency $\Delta m_{\mathrm{d}}$ is well known, we can determine the flavor of the $B_{\mathrm{REC}}$ at the instant it decayed up to a dilution factor related to the mistag probability. For $\mathrm{CP}$ eigenstate decays we construct a $\mathrm{CP}$ asymmetry as

$$
\mathscr{A}(\Delta t)=\frac{R(\Delta t)-\bar{R}(\Delta t)}{R(\Delta t)+\bar{R}(\Delta t)}=\eta_{f} S \sin \left(\Delta m_{\mathrm{d}} \Delta t\right)-C \cos \left(\Delta m_{\mathrm{d}} \Delta t\right)
$$

where $R(\bar{R})$ is the decay-rate for $B^{0}\left(\bar{B}^{0}\right)$ tagged events, and $\eta_{f}$ is the CP eigen-value of the final state. $S$ or $C$ are non-zero if the decay is $C P$ violating.

In the case of $B \rightarrow h h$ decays, the interference between $B^{0}-\bar{B}^{0}$ mixing and tree amplitudes results in $S$ being related to $\alpha$. However it is not straightforward to interpret the measurement of $S$ directly in terms of $\alpha$ as there are potential loop (penguin) contributions that complicate the issue. Nevertheless it is possible to extract constraints on $\alpha$ using $S U(2)$ isospin or $S U$ (3) flavor based relations [3].

\section{MEASUREMENTS OF $\alpha$}

Before the B-Factories started to take data the most promising way to measure $\alpha$ was thought to be through the study of $B \rightarrow \pi \pi$ decays as the $\pi^{+} \pi^{-}$state has a large branching fraction, $\mathscr{B} \sim 5.2 \times 10^{-6}$. The branching fractions of $B \rightarrow \pi^{ \pm} \pi^{0}$ and $\pi^{0} \pi^{0}$ are required to constrain penguin contributions in this decay. The branching fraction for $\pi^{ \pm} \pi^{0}$ is comparable to that for $\pi^{+} \pi^{-}$, however the branching fraction of $\pi^{0} \pi^{0}$ is $1.6 \times 10^{-6}$ which turns out to be neither small enough, nor large enough to enable us to strongly constrain penguin pollution in these decays. We now know that there is a significant penguin contamination in the $\pi^{+} \pi^{-}$mode. Nevertheless useful constraints on $\alpha$ have been obtained as a result of experimental efforts over the past decade [4].

Historically the BABAR and Belle measurements of $S$ and $C$ in $B \rightarrow \pi^{+} \pi^{-}$have not been in good agreement, however in recent years the compatibility between results has improved. Today the results of the two B-Factories are in agreement with each other giving a world average measurement of $S=-0.65 \pm 0.07$ and $C=-0.38 \pm 0.06$. The constraint obtained on $\alpha$ is limited by our ability to determine the penguin contribution and the region $20^{\circ} \leq \alpha \leq 70^{\circ}$ has been excluded at more than $3 \sigma$ using $B \rightarrow \pi \pi$ decays.

The method of measuring $\alpha$ using $B \rightarrow \rho \rho$ decays is similar to that used for $B \rightarrow \pi \pi$ decays. The main differences are that $\rho \rho$ decays have a smaller signal to background ratio, and the final state is a $\mathrm{CP}$ admixture, where the dominant $\mathrm{CP}$ even signal component has to be identified in order to extract time-dependent information related to $\alpha$. The 
dominant CP even signal component is extracted from a maximum-likelihood fit that accounts for the presence of longitudinal and transverse components. The fraction of longitudinally polarized (CP even) signal is close to unity, which simplifies this process.

The precision of the constraint on $\alpha$ obtained using $\rho \rho$ decays has changed over time due to the constraints placed on penguin amplitudes. The initial measurements from $B A B A R$ benefited from a large measured branching fraction of $B^{ \pm} \rightarrow \rho^{ \pm} \rho^{0}$ [5]. This branching fraction is crucial for the determination of the penguin contribution. The result of measuring a large branching fraction for $\rho^{ \pm} \rho^{0}$ is a 4-fold degenerate solution in $\alpha$ with flat isospin triangles. In 2007 the measured branching fraction for $B^{ \pm} \rightarrow \rho^{ \pm} \rho^{0}$ was reduced, resulting in a larger error on $\alpha$. The latest $\rho^{ \pm} \rho^{0}$ branching fraction measurement has increased the world average, which in turn decreases the penguin pollution, and overall error on alpha [7, 8]. The current constraint is $\alpha=\left(92.4_{-6.5}^{+6.0}\right)^{\circ}$ using the Gronau-London $S U(2)$ approach. This is now in good agreement with the $S U$ (3) approach from Beneke et al. which gives $\alpha=\left(89.8_{-6.4}^{+7.0}\right)^{\circ}$ [7]. The difference in central value of these two interpretations comes from neglecting a small correction from electroweak loop contributions in the Isospin analysis. Recent searches for $B^{0} \rightarrow \rho^{0} \rho^{0}$ have resulted in $B A B A R$ seeing evidence for a signal, whereas Belle are currently unable to confirm the existence of this channel [8]. The BABAR analysis included a timedependent measurement of $S$ and $C$. It would be possible to reduce the number of ambiguities in the isospin analysis of $\rho \rho$ decays with a precision measurement of $S$ and $C$ for $B \rightarrow \rho^{0} \rho^{0}$.

One important set of measurements that has been performed is the constraint on $\alpha$ using $B \rightarrow \rho \pi\left(\pi^{+} \pi^{-} \pi^{0}\right)$ decays [9]. While the constraint on $\alpha$ obtained through these measurements is not as strong as that from $\rho \rho$ decays, it should be noted that a high precision update of this mode will be instrumental in resolving ambiguities inherent in the measurement of $\alpha$.

In addition to the aforementioned decays, there are a number of other decay modes that may provide useful measurements of $\alpha$ in the future. These include $B$ meson decays to final states with axial-vector mesons such as $a_{1} \pi, a_{1} \rho, a_{1} a_{1}, b_{1} \pi$, and $b_{1} \rho$. While it should be possible to start measuring $\alpha$ from the some of these modes with the current $B$ factories, any precision measurements would be the remit of a Super Flavor Factory.

\section{SUMMARY}

The UTfit [10] and CKM fitter [11] groups combine information on $\alpha$ using different statistical methods. These groups report average values of $\alpha$ using all measurements as $(92.0 \pm 3.4)^{\circ}$, and $\left(90.6_{-4.2}^{+3.8}\right)^{\circ}$, respectively. Figure 1 shows the constraint on $\alpha$ obtained by UTfit on combining direct measurements, where $\alpha=(92 \pm 7)^{\circ}$. The corresponding constraint from CKM fitter is $\alpha=\left(89_{-4.2}^{+4.4}\right)^{\circ}$.

In summary significant progress in the measurement of $\alpha$ has been made over the last decade by the SLAC and KEK B-Factories. This provides an important independent crosscheck of the CKM paradigm describing CP violation in the SM. Future experiments such as the proposed Super Flavor Factories, SuperB in Italy and SuperKEKB in Japan will provide us with the necessary tools and data to perform precision tests of $\alpha$ in 


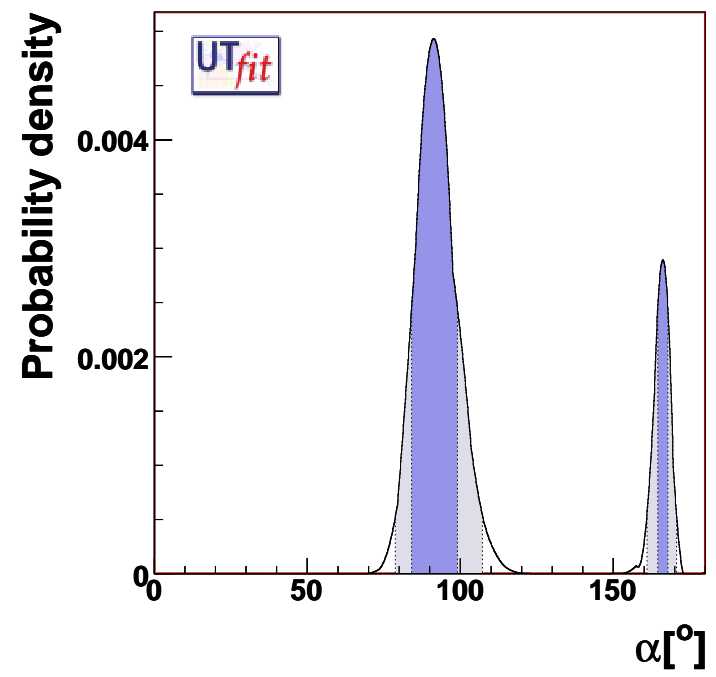

FIGURE 1. The UTfit constraint obtained on $\alpha$ using direct measurements.

individual channels. Any deviations from SM expectation measured could hint at new physics corrections to the CKM paradigm.

\section{ACKNOWLEDGMENTS}

The author would like to thank the conference organizers for the opportunity to give this talk, and the scientists working on the SLAC and KEK B-Factories for their superlative efforts. This work is supported by the UK Science and Technology Facilities Council.

\section{REFERENCES}

1. N. Cabibbo, Phys. Rev. Lett. 10, 531 (1963); M. Kobayashi and T. Maskawa, Prog. Theor. Phys. 49, 652 (1973).

2. BABAR Collaboration, Nucl. Instrum. Methods A 479, 1 (2002); Belle Collaboration, Nucl. Instrum. Methods A 479, 117 (2002).

3. M. Gronau and D. London, Phys. Rev. Lett. 65, 3381 (1990); M. Beneke et al., Phys. Lett. B 638, 68 (2006).

4. B. Aubert et al., arXiv:0807.4226, K. Abe et al. Phys. Rev. Lett. 99, 121601 (2007).

5. B. Aubert et al., Phys. Rev. Lett. 93, 231801 (2004); B. Aubert et al., Phys. Rev. Lett. 91, 171802 (2003); J. Zhang et al., Phys. Rev. Lett. 91, 221801 (2003).

6. B. Aubert et al., Phys. Rev. Lett. 102, 141802 (2009); A. Somov et al., Phys. Rev. D 76, 011104 (2007).

7. B. Aubert et al., Phys. Rev. D 76, 052007 (2007).

8. B. Aubert et al., Phys. Rev. D 78, 071104 (2008); C. C. Chaing et al., Phys. Rev. D 78, 111102 (2008).

9. B. Aubert et al., Phys. Rev. D76, 012004 (2007); A. Kusaka et al., Phys. Rev. D 77, 072001 (2008).

10. The UTfit Collaboration, M. Bona et al., http://www. utfit.org/.

11. The CKM fitter Collaboration, J. Charles et al., http://ckmfitter.in2p3.fr/ 